\title{
Synthesis And Biological Evaluation Of Novel 5-Methyl-1, 4- Disubstituted 1h-1, 2, 3-Triazole Derivatives
}

\author{
A. Pavani ${ }^{1}$, T.Lakhshmi Viveka ${ }^{2}$ \\ ${ }^{I}$ G. Pulla Reddy College of pharmacy, Mehdipatnam, Hyderabad-500028 \\ ${ }^{2}$ Natco Research Centre, Sanath Nagar, Hyderabad-500018
}

\begin{abstract}
Six triazole derivatives have been synthesized using $p$-toludine and $p$ - anisidine as starting materials. Diazotization in presence of $\mathrm{NaN}_{3}$ and followed by cyclisation esterification with absolute methanol and followed by reduction with $\mathrm{NaBH}_{4}$ and oxidation with $\mathrm{NaOCl}$ and $\mathrm{NaBr}$ in presence of 2, 2, 6, 6tetramethyl-1-piperinyloxy free radical (TEMPO) gave 5-methyl-1-(4-methyl phenyl)-1H-1, 2, 3-triazole-4carbaldehyde and 5-methyl-1-(4-methoxy phenyl)-1H-1, 2, 3-triazole-4-carbaldehyde (5a \& 5b). Finally, compounds $5 a$ and $5 b$ on condensation with primary amines like cyclohexyl amine, (+) phenyl ethyl amine, (-) phenyl ethyl amine using sodium triacetoxy borohydride gave 5-methyl-1, 4- disubstituted-1H-1, 2, 3-triazole derivatives $(6 a-f)$.

All the compounds were characterized by physical (melting point and TLC) and spectral data (IR, Mass, ${ }^{1}$ HNMR). The synthesized compounds were screened for antibacterial and anti-inflammatory activities. Some of the compounds were found to possess mild antibacterial activity at the tested concentrations. In antiinflammatory study, compounds $(6 a, 6 b, 6 d$, and $6 e)$ showed good activity when compared with that of standard drug ibuprofen. Therefore these compounds can be further exploited to get the lead compound.
\end{abstract}

Key Words: Triazole, sodium triacetoxy borohydride, carbaldehydes, amines, antibacterial, anti-inflammatory.

\section{Introduction}

Triazoles are an important class of bioactive molecules having a wide spectrum of biological activities. A large number of 1, 2, 3-triazole derivatives have been reported to be synthetic intermediates and pharmaceuticals $^{2-3}$. Several therapeutically interesting 1, 2, 3-traizoles have been reported, including anti-HIV agents $^{4-7}$, antimicrobial compounds ${ }^{8}$, selective adrenergic receptor agonists ${ }^{9}$, kinase inhibitors ${ }^{10-11}$, and other enzyme inhibitors ${ }^{12-13}$.These observations prompted us to synthesize the title compounds with presumption that incorporation of amines and triazole nuclei would produce new compounds with significant antibacterial and anti-inflammatory activity.

\section{Anti microbial activity:}

The synthesized compounds were tested for their antibacterial activity by cup plate method by measuring the zone of inhibition on agar plates in mm against gram-positive bacteria (Staphylococcus aureus and Bacillus subtilis) and two gram negative bacteria (Escherichia coli and Pseudomonas aureginosa) at a concentration of $50 \mu \mathrm{l}$ using Ciprofloxacin and Ampicillin as standard drugs and DMSO as a control. The screening results of the compounds indicated that all the compounds exhibited mild to moderate activity. Antifungal activity of the synthesized compounds was performed by turbidimetric method against two fungi (Candida albicans, and Saccharomyces cerevisiae) using Ketoconazole as a standard drug and DMSO as control where the compounds showed very least activity.

\section{Anti inflammatory activity:}

All the synthesized compounds were screened for anti-inflammatory activity by carrageen an induced rat paw edema method of winter et al ${ }^{14}$.Ibuprofen was used as a standard. From the data obtained, the mean edema volume and percentage reduction in edema was calculated. Looking at the anti-inflammatory activity, compounds $6 a, 6 b, 6 d, 6 e$ have significant anti-inflammatory activity and the results are comparable to that of standard ibuprofen

\section{Materials And Methods:}

Melting points were determined using open capillary tubes on ANALAB melting point apparatus and are uncorrected. Completion of the reactions were monitored from time to time by analytical TLC using EMerck silica gel $F_{254}, 0.25 \mathrm{~mm}$ precoated aluminium plates and visualized under UV light $(254 \mathrm{~nm})$. The purity of the compounds was checked by single spot in TLC and the solvent system for the TLC was determined by trial and error basis. All the IR spectra were recorded on SHIMADZU FT-IR spectrophotometer by using $1 \% \mathrm{KBr}$ 
discs. All the ${ }^{l} H$ NMR spectra have been recorded in $\mathrm{CDCl}_{3}$ solvent unless otherwise mentioned. ${ }^{l} \mathrm{H} N \mathrm{~N} R$ Chemical shifts are reported on Brucker $400 \mathrm{MHz}$ relative to tetra methyl silane as internal standard in delta scale. Mass spectra of the compounds were recorded on mass spectrophotometer (Aligent 1100series; EI/ES$M S)$.

General procedure:

III. Results And Discussion:

Synthesis of 5-methyl-1-(4-methyl phenyl)-1 $\mathrm{H}$-1,2,3-triazole-4-carbaldehyde (5a)from 5-methyl1-(4-methyl phenyl)-1H-1,2,3-triazole-4-yl) methanol

$1.5 \mathrm{~g}$ (0.007 moles) of 5-methyl-1-(4-methyl phenyl)-1H-1,2,3-triazole-4-yl) methanol, $0.810 \mathrm{~g}(0.0078$ moles) of sodium bromide, $1.88 \mathrm{~g}(0.022$ moles) of sodium bicarbonate, $0.001 \mathrm{~g}$ (0.0000765 moles) of TEMPO, $15 \mathrm{ml}$ methylene chloride and $7.5 \mathrm{ml}$ water were taken into a round bottom flask. Reaction mass cooled to 10 ${ }^{0} \mathrm{C}$. To this $0.71 \mathrm{~g}$ (0.00084 moles) of sodium hypochlorite was added and reaction mass was maintained below $10{ }^{\circ} \mathrm{C}$. after reaction completion isolate the product, filtered and washed with heptane and dried at $40{ }^{\circ} \mathrm{C}$ under vacuum to yield a crystalline solid.

Synthesis of 5-methyl-1-(4-methoxy phenyl)-1H-1, 2, 3-triazole-4-carbaldehyde (5b) from 5methyl-1-(4-methoxy phenyl)-1H-1, 2, 3-triazole-4-yl) methanol

By following the above procedure, 5-methyl-1-(4-methoxy phenyl)-1H-1,2,3-triazole-4-carbaldehyde (5b) has also been prepared using (0.0066 moles )of 5-methyl-1-(4-methoxy phenyl)-1H-1,2,3-triazole-4-yl) methanol, (0.0067 moles )of sodium bromide, (0.0193 moles ) of sodium bicarbonate, (0.000066 moles) of TEMPO, $15 \mathrm{ml}$ methylene chloride and $7.5 \mathrm{ml}$ water and (0.0072 moles) of sodium hypochlorite.

Note: TEMPO is 2, 2, 6, 6-tetramethyl-1-piperrinoyloxy free radical on silica gel. It is a recyclable catalyst in oxidation of alcohols

Synthesis of 5-methyl-1,4- disubstituted-1H-1, 2, 3-triazole derivatives with primary amines:

$0.5 \mathrm{~g}(0.00213 \mathrm{moles})$ of primary amine dissolved in $6 \mathrm{ml}$ of methylene chloride was taken in a round bottom flask. To this $0.65 \mathrm{~g}$ (0.00307 moles) of sodium triacetoxy borohydride and $0.33 \mathrm{~g}$ (0.00549 moles) glacial acetic acid were added and stirred for $10 \mathrm{~min}$. To the above mixture $0.5 \mathrm{~g}$ (0.00223 moles) 5-methyl-1(4-methyl phenyl)-1H-1,2,3-triazole-4-carbaldehyde dissolved in methylene chloride was added dropwise and maintained for $4 \mathrm{~h}$ at room temperature. The progress of the reaction was monitored by TLC. At the end of the reaction, the mixture was basified with $10 \%$ aqueous potassium carbonate solution and extracted with methylene chloride. The extract was washed with brine solution, concentrated and purified by column chromatography.

Following the above procedure, 5-methyl-1-(4-methoxy phenyl)-1H-1,2,3-triazole derivatives (6d),(6e) and (6f) has also been prepared using 0.00223 moles of 5-methyl-1-(4-methoxy phenyl)-1H-1,2,3-triazole-4carbaldehyde.

Analytical data of the above compounds:

$\mathrm{N}$-\{[5-methyl-1-(4-methylphenyl)-1H-1,2,3-triazole-4yl]methyl\}methyl cyclohexanamine (6a): This compound was obtained as white solid. $\boldsymbol{m p} 190-192^{\circ} \mathrm{C} ; \boldsymbol{I R}: 1592 \mathrm{~cm}^{-1}(N=N=N H$ stretch $), 3445.4 \mathrm{~cm}^{-1}$ (NH stretch), $1655.2 \mathrm{~cm}^{-1}$ (N-H bend), $1349.9 \mathrm{~cm}^{-1},\left(C-N\right.$ stretch); Mass (m/z): $285(M+1) ;{ }^{1} \boldsymbol{H}$ NMR $\left(\mathrm{CDCl}_{3}, 400 \mathrm{Mhz}\right): 1.1-1.197(\mathrm{~m}, 2 \mathrm{H}) ; 1.226-1.334(\mathrm{~m}, 2 \mathrm{H},) ; 1.62-1.65(\mathrm{~m}, \mathrm{lH}) ; 1.70(\mathrm{bs}, 2 \mathrm{H}) ; 1.74-1.77(\mathrm{~m}$, $2 H) ; 1.94-1.97(m, 2 H) ; 2.29(s, 3 H), 2.44(s, 3 H) ; 2.53-2.58(\mathrm{~m}, 1 \mathrm{H}) ; \quad 3.89(\mathrm{~s}, 2 \mathrm{H}), 7.32(\mathrm{~s}, 4 \mathrm{H})$.

$\mathrm{N}$-\{[5-methyl-1-(4-methylphenyl)-1H-1,2,3-triazole-4yl]methyl\}methyl\}-1-(+)phenyl ethanamine (6b):This compound was obtained as pale yellow solid. mp 195-198 ${ }^{\circ}$; IR :1592 $\mathrm{cm}^{-1}$ (N=N=NH stretch),

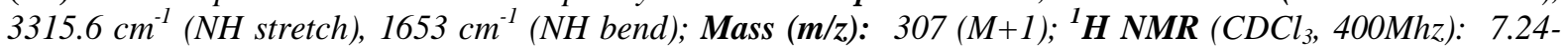
$7.45(\mathrm{~m}, 12 \mathrm{H}, \mathrm{Ar}-\mathrm{H}), 3.85-3.91(\mathrm{~m}, \mathrm{lH}), 3.66-3.75(\mathrm{~m}, 2 \mathrm{H}), 2.44(\mathrm{~s}, 3 \mathrm{H}), 2.41(\mathrm{~s}, \mathrm{NH}), 2.15(\mathrm{~s}, 3 \mathrm{H})$

\section{N-\{[5-methyl-1-(4-methylphenyl)-1H-1,2,3-triazole-4yl]methyl\}methyl\}-1(-)phenyl}

ethanamine(6c): This compound was obtained as pale yellow solids.mp:196-198 ${ }^{\circ}$; IR: $3297.3 \mathrm{~cm}^{-1}(\mathrm{NH}$ stre) $1654 \mathrm{~cm}^{-1}(\mathrm{NH}$ bend); $\operatorname{mass}(\mathrm{m} / \mathrm{z}): 307(\mathrm{M}+1)$.

$\mathrm{N}$-\{[5-methyl-1-(4-methoxyphenyl)-1H-1,2,3-triazole-4yl]methyl $\}$ methyl cyclohexanamine (6d): This compound was obtained as pale yellow solids.mp:201-203 ${ }^{0}$; IR: $1591 \mathrm{~cm}^{-1}(N=N=N H$ 
stretch), $3394 \mathrm{~cm}^{-1}$ (NH stretch), $1654 \mathrm{~cm}^{-1}$ (N-H bend), $1349.9 \mathrm{~cm}^{-1}$ (C-N stretch); Mass $(\mathrm{m} / \mathrm{z}): 301(M+1)$;

(1R)-N-\{[5-methyl-1-(4-methoxyphenyl)-1H-1,2,3-triazole-4yl]methyl\}methyl\}-1-phenyl

ethanamine (6e) :This compound was obtained as white solid.mp: $210^{\circ} \mathrm{C}$; IR: $3445.5 \mathrm{~cm}^{-1}$ (NH stretch), 1654 $\mathrm{cm}^{-1}$ (NH bend); Mass(m/z):323 (M+1); ${ }^{1} \boldsymbol{H} \mathbf{N M R}\left(\mathrm{CDCl}_{3}, 400 \mathrm{Mhz}\right): 1.88-1.89\left(\mathrm{~d}, 3 \mathrm{H}, \mathrm{CH}_{3}\right), 2.36(\mathrm{~s}, 3 \mathrm{H}, \mathrm{Ar}-\mathrm{H})$, $3.87\left(\mathrm{~s}, 3 \mathrm{H}, \mathrm{OCH}_{3}\right), 3.99-4.167\left(\mathrm{~s}, 2 \mathrm{H}, \mathrm{CH}_{2}\right), 7.00-7.22(\mathrm{~s}, 2 \mathrm{H}, \mathrm{Ar}-\mathrm{H}), 7.33-7.48(\mathrm{~m}, 3 \mathrm{H}, \mathrm{Ar}-\mathrm{H}), 7.82-7.84(d$, $2 \mathrm{H}, \mathrm{Ar}-\mathrm{H}), 10.17(\mathrm{~s}, \mathrm{lH}, \mathrm{Ar}-\mathrm{H}), 10.38(\mathrm{~s}, 1 \mathrm{H}, \mathrm{Ar}-\mathrm{H})$

(1S)-N-\{[5-methyl-1-(4-methoxyphenyl)-1H-1， 2, 3-triazole-4yl]methyl\}methyl\}-1-phenyl ethanamine (6f): This compound was obtained as white solid. IR : $1591.3 \mathrm{~cm}^{-1}(N=N=N H$ stretch), $3298.4 \mathrm{~cm}^{-1}$ (NH stretch), $1654.2 \mathrm{~cm}^{-1}$ (NH bend); Mass (m/z): 323(M+1); ${ }^{1} \boldsymbol{H} \mathrm{NMR}\left(\mathrm{CDCl}_{3}, 400 \mathrm{Mhz}\right): 7.00$ 7.031 (2H, $m, \mathrm{Ar}-\mathrm{H}), 7.278(2 \mathrm{H}, \mathrm{d}, \mathrm{Ar}-\mathrm{H}), 7.31-7.429$ (5H, $\mathrm{m}, \mathrm{Ar}-\mathrm{H}), 1.40-1.44\left(3 \mathrm{H}, \mathrm{m}, \mathrm{CH}_{3}\right), 0.93(\mathrm{~s}, \mathrm{H}, \mathrm{CH})$, 3.87-3.92 (t, 3H, $\left.\mathrm{CH}_{3}\right), 2.26\left(\mathrm{~s}, 3 \mathrm{H}, \mathrm{OCH}_{3}\right), 1.40-1.44\left(\mathrm{t}, 3 \mathrm{H}, \mathrm{CH}_{3}\right)$

TABLE 1

Physical data of 5-methyl-1, 4-disubstituted-1H-1,2,3-triazoles

\begin{tabular}{|c|c|c|c|c|}
\hline S.NO & Compd & STRUCTURE & M. $P\left({ }^{0} C\right)$ & Yield (\%) \\
\hline 1 & $6 a$ & & 192 & 72 \\
\hline 2 & $6 b$ & & 198 & 69 \\
\hline 3 & $6 c$ & & 197 & 70 \\
\hline 4 & $6 d$ & & 203 & 68 \\
\hline 5 & $6 e$ & & 210 & 65 \\
\hline 6 & $6 f$ & & 208 & 72 \\
\hline
\end{tabular}




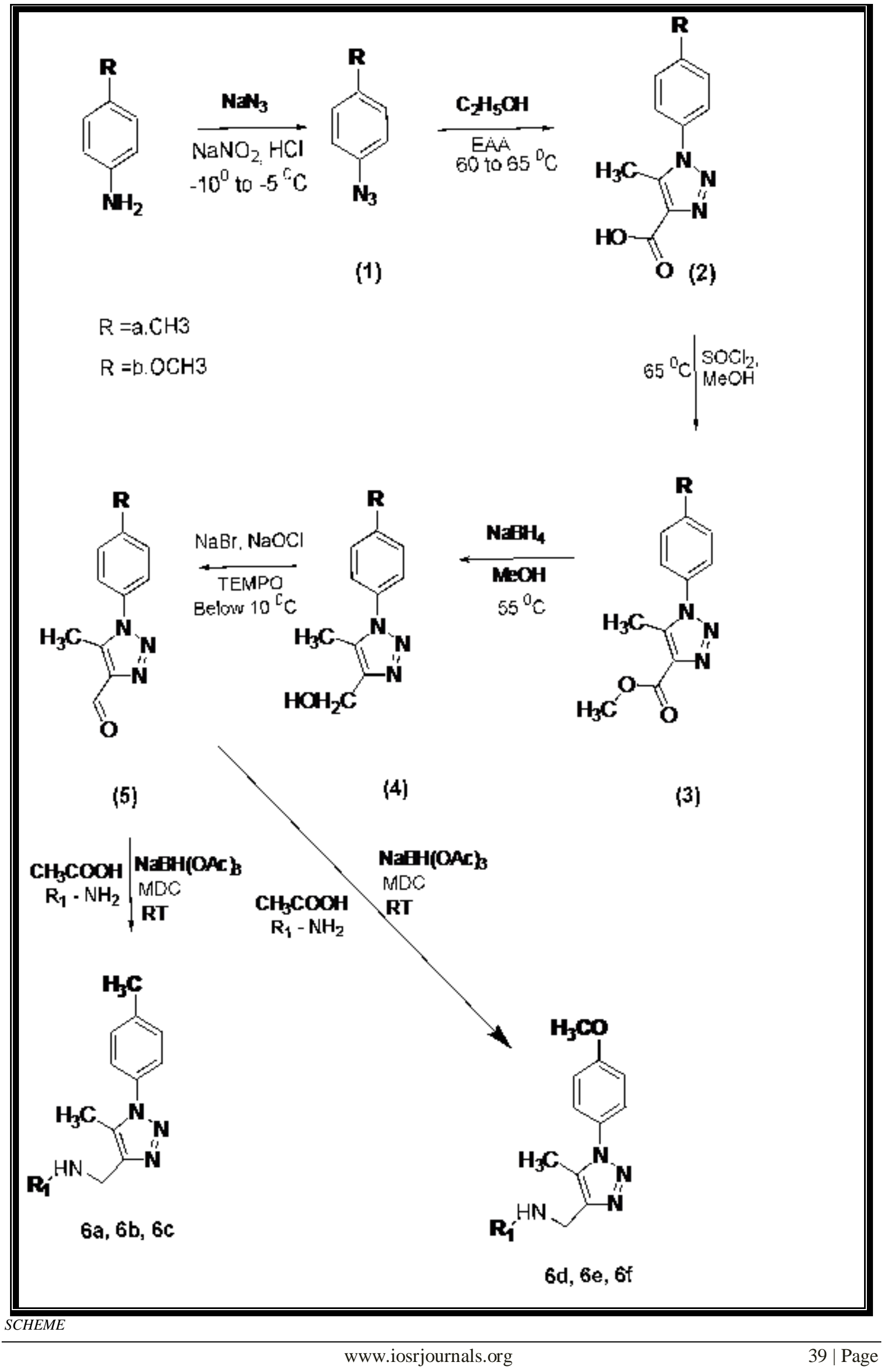


TABLE 2: Anti-bacterial activity of 5-methyl-1,4-disubstituted 1H-1,2,3-triazole derivatives

\begin{tabular}{|c|c|c|c|c|c|}
\hline \multirow[t]{2}{*}{ compound } & \multirow[t]{2}{*}{$R$} & \multicolumn{4}{|c|}{ Zone of inhibition } \\
\hline & & $\begin{array}{l}\text { Bacillus } \\
\text { subtilis(+ve) } \\
\text { mm }\end{array}$ & $\begin{array}{l}\text { Staphylococcus } \\
\text { aureas }(+v e) \\
\mathrm{mm}\end{array}$ & $\begin{array}{l}\text { Escheresia } \\
\text { coli(-ve) } \\
\mathrm{mm}\end{array}$ & $\begin{array}{l}\text { Pseudomonas } \\
\text { aeruginosa } \\
\mathrm{mm}\end{array}$ \\
\hline $6 a$ & $\mathrm{CH} 3$ & 16 & 12 & $N A$ & $N A$ \\
\hline $6 b$ & $\mathrm{CH} 3$ & 18 & 20 & $N A$ & $N A$ \\
\hline $6 d$ & $\mathrm{OCH} 3$ & $N A$ & 12 & $N A$ & 10 \\
\hline $6 e$ & $\mathrm{OCH} 3$ & 10 & 12 & $N A$ & 11 \\
\hline $\begin{array}{l}\text { Ciprofloxacin } \\
(10 \mu g / 50 \mu l)\end{array}$ & --- & \multicolumn{2}{|l|}{38} & \multicolumn{2}{|l|}{29} \\
\hline
\end{tabular}

TABLE 3: Anti-inflammatory activity of 5-methyl-1, 4-disubstituted 1H-1, 2, 3-triazole derivatives mean paw edema volume (ml)

\begin{tabular}{|l|l|l|l|l|l|l|}
\hline Treatment & $\begin{array}{l}\text { Dose } \\
(\mathrm{mg} / \mathrm{kg})\end{array}$ & $30 \mathrm{~min}$ & $1 \mathrm{~h}$ & $2 \mathrm{~h}$ & $3 \mathrm{~h}$ & $4 \mathrm{~h}$ \\
\hline Control & 100 & $0.28 \pm 0.014$ & $0.39 \pm 0.025$ & $0.60 \pm 0.047$ & $0.82 \pm 0.013$ & $0.82 \pm 0.029$ \\
\hline $\begin{array}{l}\text { Standard } \\
\text { (Ibuprofen) }\end{array}$ & 100 & $0.18 \pm 0.045$ & $0.20 \pm 0.059$ & $0.22 \pm 0.06$ & $0.17 \pm 0.038$ & $0.25 \pm 0.043$ \\
\hline 6a & 100 & $0.22 \pm 0.057$ & $0.24 \pm 0.038$ & $0.28 \pm 0.014$ & $0.33 \pm 0.029$ & $0.31 \pm 0.029$ \\
\hline 6b & 100 & $0.20 \pm 0.043$ & $0.22 \pm 0.047$ & $0.26 \pm 0.050$ & $0.29 \pm 0.015$ & $0.30 \pm 0.030$ \\
\hline 6c & 100 & $0.21 \pm 0.013$ & $0.23 \pm 0.056$ & $0.33 \pm 0.048$ & $0.42 \pm 0.018$ & $0.50 \pm 0.038$ \\
\hline 6d & 100 & $0.21 \pm 0.027$ & $0.23 \pm 0.053$ & $0.27 \pm 0.035$ & $0.34 \pm 0.026$ & $0.31 \pm 0.058$ \\
\hline 6e & 100 & $0.20 \pm 0.048$ & $0.21 \pm 0.025$ & $0.25 \pm 0.053$ & $0.24 \pm 0.052$ & $0.29 \pm 0.052$ \\
\hline 6f & 100 & $0.22 \pm 0.031$ & $0.24 \pm 0.022$ & $0.34 \pm 0.034$ & $0.43 \pm 0.022$ & $0.49 \pm 0.026$ \\
\hline
\end{tabular}

Edema volume $=$ mean \pm SEM

TABLE 4: Percentage protection against edema formation:

\begin{tabular}{|l|l|l|l|l|l|l|l|}
\hline Treatment & $\begin{array}{l}\text { Dose } \\
(\mathrm{mg} / \mathrm{kg})\end{array}$ & $R$ & $30 \mathrm{~min}$ & $1 \mathrm{~h}$ & $2 \mathrm{~h}$ & $3 \mathrm{~h}$ & $4 h$ \\
\hline $\begin{array}{l}\text { Standard } \\
\text { (Ibuprofen })\end{array}$ & 100 & ---- & $35.71 \%$ & $48.71 \%$ & $63.33 \%$ & $79.26 \%$ & $69.51 \%$ \\
\hline $6 a$ & 100 & $\mathrm{CH3}$ & $21.42 \%$ & $38.46 \%$ & $53.33 \%$ & $59.75 \%$ & $59.75 \%$ \\
\hline $6 b$ & 100 & $\mathrm{CH3}$ & $28.57 \%$ & $43.58 \%$ & $56.66 \%$ & $64.63 \%$ & $63.41 \%$ \\
\hline $6 c$ & 100 & $\mathrm{CH3}$ & $25.00 \%$ & $41.02 \%$ & $45.06 \%$ & $48.78 \%$ & $39.90 \%$ \\
\hline $6 d$ & 100 & OCH3 & $25.00 \%$ & $41.02 \%$ & $55.00 \%$ & $58.53 \%$ & $62.19 \%$ \\
\hline $6 e$ & 100 & OCH3 & $28.57 \%$ & $46.15 \%$ & $58.33 \%$ & $70.73 \%$ & $64.63 \%$ \\
\hline $6 f$ & 100 & OCH3 & $21.42 \%$ & $38.46 \%$ & $43.33 \%$ & $47.54 \%$ & $38.82 \%$ \\
\hline
\end{tabular}

\section{Conclusion:}

Among the compounds tested (6a) and (6b) exhibited mild activity against Bacillus subtillis , Staphylococcus aureus and compounds (6d), (6e) exhibited mild activity against Staphylococcus aureus and Pseudomonas aeruginosa at a concentration of $1000 \mu \mathrm{g} / 50 \mu \mathrm{l}$ compared with the standard drug ciprofloxacin $(10 \mu \mathrm{g} / 50 \mu \mathrm{l})$.None of the compounds have shown any activity against fungal strains Candida albicans and Saccharomyces cerevisiae at a concentration of $1000 \mu \mathrm{g} / 50 \mu 1$.In anti-inflammatory screening the compounds (6a),(6b),(6d),(6e) have shown good anti-inflammatory activity at a concentration of $100 \mathrm{mg} / \mathrm{kg}$ compared with the standard drug ibuprofen $(100 \mathrm{mg} / \mathrm{kg})$. Therefore, these compounds can be further exploited to get lead compounds.

\section{Acknowledgement:}

The authors were thankful to NATCO pharma limited, Hyderabad and to the management of G.Pulla Reddy College of Pharmacy, Hyderabad for providing facilities. 


\section{References}

[1] Theophil Eicher, Siegfried Hauptmann, The chemistry of heterocyclic, second edition, pg: 200

[2] Fan W.Q.; Katritzky, A.R. 1,2,3 Triazoles. In Comprehensive Heterocyclic Chemistry II; Katritzky, A.R., Rees, C.W., Scriven, E.F.V., Eds.; Pergamon Press: New York, NY, USA, 1996; Vol. 4. pp. 1-126

[3] Katritzky, A.R.; Zhang, Y.; Singh, S.K. 1,2,3-Triazole formation under mild conditions via 1,3-dipolar cycloaddition of acetylenes with azides. Heterocycles 2003, 60, 1225-1239

[4] Alvarez, R.; Velazquez, S.; San, R.; Aquaro, S.; De, C.E.; Perno, C.F.; Karlsson, A.; Balzarini, J.; Camarasa, M.J. 1,2,3-Triazole[2',5'-bis-O-(tert-butyldimethylsilyl)-beta-D-ribofuranosyl]-3'-spiro-5"-(4"-amino-1",2"-oxathiole 2",2"-dioxide) (TSAO) analogues: Synthesis and anti-HIV-1 activity. J. Med. Chem. 1994, 37, 4185-4194

[5] Velazquez, S.; Alvarez, R.; Perez, C.; Gago, F.; De C.E.; Balzarini, J.; Camarasa, J.M. Regiospecific synthesis and anti-human immunodeficiency virus activity of novel 5-substituted Nalkylcarbamoyl and N,N-dialkylcarbamoyl 1,2,3-triazole-TSAO analogues. Antiviral Chem.Chemother. 1998, 9, 481-489.

[6] Whiting, M.; Muldoon, J.; Lin, Y.C.; Silverman, S.M.; Lindstrom, W.; Olson, A.J.; Kolb, H.C.; Finn, M.G.; Sharpless, B.K.; Elder, J.H.; Fokin, V.V. Inhibitors of HIV-1 protease by using in situ click chemistry. Angew. Chem. Int. Ed. 2006, 45, 1435-1439

[7] Brik, A.; Muldoon, J.; Lin, Y.C.; Elder, J.C.; Goodsell, D.S.; Olson, A.J.; Fokin, V.V.; Sharpless, B.K.; Wong, C.H. Rapid diversityoriented synthesis in microtiter plates for in situ screening of HIV protease inhibitors. Chem. Bio. Chem. 2003, 4, 1246-1248

[8] Genin, M.J.; Allwine, D.A.; Anderson, D.J.; Barbachyn, M.R.; Emmert, D.M.; Garmon, S.A.; Graber, D.R.; Grega, K.C.; Hester, J.B.; Hutchinson, D.K.; Morris, J.; Reischer, R.J.; Ford, C.W.; Zurenko, G.E.; Hamel, J.C.; Schaadt, R.D.; Stapert,D.; Yagi, B.H. Substituent effects on the antibacterial activity of nitrogen-carbon-linked (azolylphenyl) oxazolidinones with expanded activity against the fastidious gram-negative organisms Haemophilus influenza and Moraxella catarrhalis. J. Med. Chem. 2000, 43, 953-97

[9] Brockunier, L.L.; Parmee, E.R.; Ok, H.O.; Candelore, M.R.; Cascieri, M.A.; Colwell, L.F.; Deng, L.; Feeney, W.P.; Forrest, M.J.; Hom, G.J.; MacIntyre, D.E.; Wyvratt, M.J.; Fisher, M. H.; Weber, A.E. Human beta 3 -adrenergic receptor agonists containing 1,2,3triazole-substituted benzenesulfonamides. Bioorg. Med. Chem. Lett. 2000, 10, 2111-2114

[10] Pande, V.; Ramos, M.J. Structural basis for the GSK-3 beta binding affinity and selectivity against CDK-2 of 1-(4-aminofurazan3yl)-5-dialkylaminomethyl-1H-[1,2,3] triazole-4-carboxylic acid derivatives. Bioorg. Med. Chem. Lett. 2005, 15, 5129-5135

[11] Olesen, P.H.; Sorensen, A.R.; Urso, B.; Kurtzhals, P.; Bowler, A.N.; Ehrbar, U.; Hansen, B.F. Synthesis and in vitro characterization of 1-(4-Aminofurazan-3-yl)-5-dialkylaminomethyl-1H-[1,2,3]triazole-4-carboxylic acid derivatives. A new class of selective GSK-3 Inhibitors. J. Med.Chem. 2003, 46, 3333-3341

[12] Krasinski, A.; Radic, Z.; Manetsch, R.; Raushel, J.; Taylor, P.; Sharpless, B.K.; Kolb, H.C. In situ selection of lead compounds by click chemistry: Target-guided optimization of aceylcholinesterase inhibitors. J. Am. Chem. Soc. 2005, 127, 6686-6692

[13] Mocharla, V.P.; Colasson, B.; Lee, L.V.; Roeper, S.; Sharpless, B.K.; Wong, C.H.; Kolb, H.C. In situ click chemistry: Enzymegenerated inhibitors of carbonic anhydrase II. Angew. Chem. Int. Ed. 2005, 44, 116-120

[14] A. Winter, E.A.Risleyand, G.W.Nuss, Proc.Soc.Exp.Bio.Med. 111(1962), pp.544. 